\title{
217. A Class of Markov Processes with Interactions. II
}

\author{
By Tadashi Ueno \\ Department of Mathematics, Faculty of General Education, \\ University of Tokyo
}

(Comm. by Zyoiti Suetuma, M. J. A., Dec. 12, 1969)

Here, we look at the branches which describe the interactions between particles of the model in [4]. This leads to finer proofs of Chapman-Kolmogorov equation and the backward equation. A consistency condition holds for probabilities of events which are determined by bundles of these branches.

1. To consider the simplest model with binary interactions, let $q(t, y) \equiv q_{1}(t, y)$ and $q_{0} \equiv q_{2} \equiv q_{3} \equiv \cdots \equiv 0$, and write $\pi\left(y^{\prime} \mid t, y, E\right)$ for $\pi_{1}\left(y_{1} \mid t, y, E\right)$ in 1 of [4]. ${ }^{1)}$ Then, the forward and the backward equations are

$$
\begin{aligned}
& P^{(f)}(s, x, t, E)=P_{0}(s, x, t, E)+\int_{s}^{t} d \tau \int_{R^{2}} P^{(f)}(s, x, \tau, d y) \\
& \times P_{s, \tau}^{(f)}\left(d y^{\prime}\right) q(\tau, y) \int_{R} \pi\left(y^{\prime} \mid \tau, y, d z\right) P_{0}(\tau, z, t, E), \\
& P^{\left(P_{s o s}^{(f)}\right)}(s, x, t, E)=P_{0}(s, x, t, E)+\int_{s}^{t} d \tau \int_{R^{2}} P_{0}(s, x, \tau, d y) \\
& \times P_{s_{0 \tau}}^{(f)}\left(d y^{\prime}\right) q(\tau, y) \int_{R} \pi\left(y^{\prime} \mid \tau, y, d z\right) P^{\left(P_{s_{0} \tau}^{(f)}\right)}(\tau, z, t, E),
\end{aligned}
$$

where $P_{s, \tau}^{(f)}(E) \doteq \int_{R} f(d x) P^{(f)}(s, x, \tau, E), \quad s_{0} \leq s \leq t$.

Let $T$ be the set of all branches which grow downward with binary branching points and the trivial branch (or a pole) $b_{0}$. For $b_{1}$ and $b_{2}$ in $T, b=\left(b_{1}, b_{2}\right)$ is the branch which has $b_{1}$ and $b_{2}$ on the left and the right side of the highest branching point. Length $l(b)$ and the number of the end points $\#(b)$ are defined by

$$
\begin{aligned}
l\left(b_{0}\right) & =0, l\left(\left(b_{1}, b_{2}\right)\right)=1+\max \left(l\left(b_{1}\right), l\left(b_{2}\right)\right), \\
\#\left(b_{0}\right)=1, ~ & \#\left(\left(b_{1}, b_{2}\right)\right)=\#\left(b_{1}\right)+\#\left(b_{2}\right) .
\end{aligned}
$$

When $\#(b)=n$, let $b\left(b_{1}, \cdots, b_{n}\right)$ be the branch $b$ with branches $b_{1}, \cdots, b_{n}$ connected at the end points, with $b_{k}$ at the $k$-th end point from the left. We write $b \geq b^{\prime}$ when $b=b^{\prime}\left(b_{1}, \cdots, b_{n}\right)$. Since the branches $b_{1}, \cdots, b_{n}$ are determined

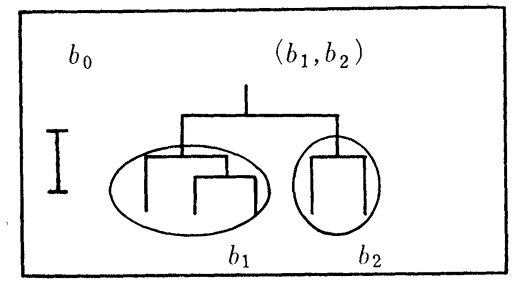

1) This is for the simplicity of descriptions. Results in this paper can be extended to the models in [4]. 


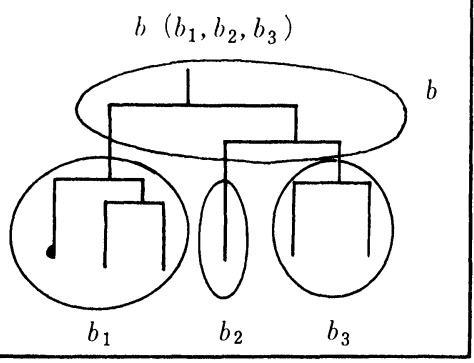

uniquely for given $b$ and $b^{\prime}(\leq b)$, we denote the bundle of branches $\left(b_{1}, \ldots\right.$ $\left.\cdots, b_{n}\right)$ by $b / b^{\prime}$. When $\#(b)=n$ and $\boldsymbol{x}=\left(x_{1}, \cdots x_{n}\right)$, let $b(\boldsymbol{x})=b\left(x_{1}, \cdots, x_{n}\right)$ be the branch $b$ with variables $x_{1}, \cdots, x_{n}$ at the end points, with $x_{k}$ at the $k$-th end point from the left. $\left(b_{1}\left(\boldsymbol{x}_{1}\right), b_{2}\left(\boldsymbol{x}_{2}\right)\right)$ and $b\left(b_{1}\left(\boldsymbol{x}_{1}\right), \cdots, b_{n}\left(\boldsymbol{x}_{n}\right)\right)$ are defined similarly.

For $b \in T, \quad x=\left(x_{1}, \cdots, x_{\sharp(b)}\right), \boldsymbol{s}=\left(s_{1}, \cdots, s_{\sharp(b)}\right)$ and $t$ such that $\max (s) \leq t$, we define $P(s, b(x), t, E)$ inductively by

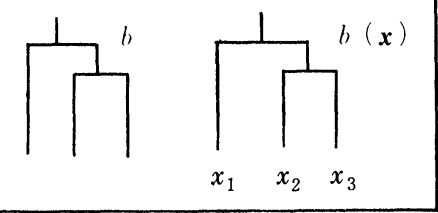

$\left.\cdots, s_{m+n}\right), \quad \boldsymbol{x}_{1}=\left(x_{1}, \cdots, x_{m}\right)$, $n=\#\left(b_{2}\right) .^{2)}$

$$
\begin{aligned}
& P\left(s_{1}, b_{0}\left(x_{1}\right), t, E\right)=P_{0}\left(s_{1}, x_{1}, t, E\right), \\
& P\left(\left(s_{1}, s_{2}\right),\left(b_{1}\left(x_{1}\right), b_{2}\left(x_{2}\right)\right), t, E\right)
\end{aligned}
$$

$$
\begin{aligned}
& =\int_{\max \left(\boldsymbol{s}_{1}, \boldsymbol{s}_{2}\right)}^{t} d \tau \int_{R^{2}} P\left(\boldsymbol{s}_{1}, b_{1}\left(\boldsymbol{x}_{1}\right), \tau, d y\right) \\
& \quad \times P\left(\boldsymbol{s}_{2}, b_{2}\left(\boldsymbol{x}_{2}\right), \tau, d y^{\prime}\right) q(\tau, y) \\
& \quad \times \int_{R} \pi\left(y^{\prime} \mid \tau, y, d z\right) P_{0}(\tau, z, t, E),
\end{aligned}
$$

where $\boldsymbol{s}_{1}=\left(s_{1}, \cdots, s_{m}\right), \boldsymbol{s}_{2}=\left(s_{m+1}, \cdots\right.$ $\boldsymbol{x}_{2}=\left(x_{m+1}, \cdots, x_{m+n}\right), \quad m=\#\left(b_{1}\right), \quad$ and Then, by a simple induction, we have

$$
P(\boldsymbol{s}, b(\boldsymbol{x}), t, R)+\int_{\max (\boldsymbol{s})}^{t} d \tau \int_{R} P(\boldsymbol{s}, b(\boldsymbol{x}), \tau, d y) q(\tau, y) \leq 1,
$$

starting with the equality in case $b=b_{0}$.

2. Theorem 1. For $s, t$, $u$ such that $\max (s) \leq t \leq u$,

$$
\begin{aligned}
P(\boldsymbol{s}, b(\boldsymbol{x}), u, E)= & \sum_{b^{\prime} \leq b} \int_{R^{\sharp}\left(b^{\prime}\right)} \prod_{b_{k} \in b^{\prime} / b^{\prime}} P\left(\boldsymbol{s}_{k}, b_{k}\left(\boldsymbol{x}_{k}\right), t, d y_{k}\right) \\
& \times P\left((t, \cdots, t), b^{\prime}(\boldsymbol{y}), u, E\right) .
\end{aligned}
$$

Note. This is an exact extension of (52) in Feller [1] to our present model:

$$
P_{n}(s, x, u, E)=\sum_{k=0}^{n} \int_{R} P_{k}(s, x, t, d y) P_{n-k}(t, y, u, E) .
$$

Outline of the proof. For $b=b_{0}$, (4) is the Chapman-Kolmogorov equation for $P_{0}(s, x, t, E)$. If we assume the result for $b_{1}$ and $b_{2}$, then for $b=\left(b_{1}, b_{2}\right)$,

$$
\begin{aligned}
& P(s, b(\boldsymbol{x}), u, E)=\left(\int_{\max (\boldsymbol{s})}^{t} d \tau+\int_{t}^{u} d \tau\right) \int_{R^{2}} P\left(s_{1}, b_{1}\left(\boldsymbol{x}_{1}\right), \tau, d y\right) \\
& \quad \times P\left(s_{2}, b_{2}(\boldsymbol{x}), \tau, d y^{\prime}\right) q(\tau, y) \int_{R} \pi\left(y^{\prime} \mid \tau, y, d z\right) P_{0}(\tau, z, u, E)
\end{aligned}
$$

2) Intuitively, $P\left(\left(s_{1}, \cdots, s_{n}\right), b\left(x_{1}, \cdots, x_{n}\right), t, E\right)$ is the probability that the particle, started at $x_{1}$ at time $s_{1}$, is in the set $E$ at time $t$ after the interactions with other particles which started at $x_{2}, \cdots, x_{n}$, at times $t_{2} \cdots, t_{n}$, respectively, where the order of the interactions are determined by the branch $b$. 


$$
\begin{aligned}
& =\int_{R} P(s, b(\boldsymbol{x}), t, d y) P_{0}(t, y, u, E)+\int_{t}^{u} d \tau \int_{R^{2}} \\
& \times\left\{\sum_{b^{\prime} \leq b_{1}} \int_{R \sharp\left(b^{\prime}\right)} \prod_{b_{k}^{\prime} \in b_{1} / b^{\prime}} P\left(\boldsymbol{s}_{k}, b_{k}^{\prime}\left(\boldsymbol{x}_{k}\right), t, d y_{k}\right) P\left((t, \cdots, t), b^{\prime}(\boldsymbol{y}), \tau, d y\right)\right\} \\
& \times\left\{\sum_{b^{\prime \prime} \leq b_{2}} \int_{\left.R \#\left(b^{\prime \prime}\right)\right)} \prod_{b_{j}^{\prime \prime} \in b_{2} / b^{\prime \prime}} P\left(\boldsymbol{s}_{j}, b_{j}^{\prime \prime}\left(\boldsymbol{x}_{j}^{\prime}\right), t, d y_{j}^{\prime}\right) P\left((t, \cdots, t), b^{\prime \prime}\left(\boldsymbol{y}^{\prime}\right) \tau, d y^{\prime}\right)\right\} \\
& \times q(\tau, y) \int_{R} \pi\left(y^{\prime} \mid \tau, y, d z\right) P_{0}(\tau, z, u, E) \\
& =\int_{R \sharp\left(b_{0}\right)} P(s, b(\boldsymbol{x}), t, d y) P\left(t, b_{0}(y), u, E\right)+\sum_{b^{\prime} \leq b_{1}} \sum_{b^{\prime} \leq b_{2}} \int_{R \sharp\left(\left(b^{\prime}, b^{\prime \prime}\right)\right)} \\
& \times \prod_{\left(b_{k}^{\prime} \in b_{1} / b^{\prime}, b_{j}^{\prime} \in b_{2} / b^{\prime \prime}\right)} P\left(\boldsymbol{s}_{k}, b_{k}^{\prime}\left(\boldsymbol{x}_{k}\right), t, d y_{k}\right) P\left(s_{j}, b_{j}^{\prime \prime}\left(\boldsymbol{x}_{j}^{\prime}\right), t, d y_{j}^{\prime}\right) \\
& \times P\left((t, \cdots, t),\left(b^{\prime}(\boldsymbol{y}), b^{\prime \prime}\left(\boldsymbol{y}^{\prime}\right)\right), u, E\right) \text {. }
\end{aligned}
$$

But, this is the right side of (4), since $b / b_{0}=\{b\}$ and there are natural one to one correspondences between $\left\{b^{\prime} \leq b_{1}\right\} \times\left\{b^{\prime \prime} \leq b_{2}\right\}$ and $\left\{b^{\prime} \leq b\right\}-\left\{b_{0}\right\}$, between $\left\{b_{1} / b^{\prime}\right\} \times\left\{b_{2} / b^{\prime \prime}\right\}$ and $b /\left(b^{\prime}, b^{\prime \prime}\right)-\left\{b_{0}\right\}$ for each fixed $b^{\prime} \leq b_{1}$ and $b^{\prime \prime} \leq b_{2}{ }^{3)}$

For a branch $b\left(\neq b_{0}\right)$ and the $i$-th end point of $b$ from the left, there is a unique pair of branches $b^{\prime}$ and $\hat{b}$ such that $b(\boldsymbol{x})=b^{\prime}\left(x_{1}, \ldots\right.$ $\left.\cdots, x_{i-1},\left(b_{0}\left(x_{i}\right), \hat{b(\hat{x})}\right), x_{k}, \cdots, x_{n}\right)$ or $b(\boldsymbol{x})=b^{\prime}\left(x_{1}, \cdots, x_{k},\left(\hat{b}(\hat{\boldsymbol{x}}), b_{0}\left(x_{i}\right)\right)\right.$, $x_{i+1}, \cdots, x_{n}$ ) for some $k$. This $\hat{b}$ is called the closest subbranch of $b$ to the $i$-th end point.

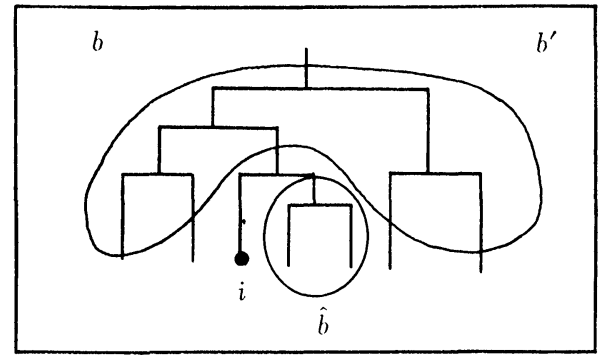

Theorem 2. By substituting $\boldsymbol{s}=\left(\boldsymbol{s}_{1}, \boldsymbol{s}_{2}\right)$ and $b(\boldsymbol{x})=\left(b_{1}\left(\boldsymbol{x}_{1}\right), b_{2}\left(\boldsymbol{x}_{2}\right)\right)$ in the place of $r_{1}$ and $y_{1}$ of $P\left(\left(r_{1}, \ldots\right.\right.$ $\left.\left.\cdots, r_{n}\right), \tilde{b}\left(y_{1}, \cdots y_{n}\right), t, E\right)$, we have

$$
\begin{aligned}
P((s, & \left.\left.r_{2}, \cdots, r_{n}\right), \tilde{b}\left(b(\boldsymbol{x}), y_{2}, \cdots, y_{n}\right), t, E\right)=\int_{\max (\boldsymbol{s}, \hat{r})}^{t} d \tau \\
\quad & \times \int_{R^{2}} P\left(s_{1}, b_{1}\left(\boldsymbol{x}_{1}\right), \tau, d y\right) P\left(s_{2}, b_{2}\left(\boldsymbol{x}_{2}\right), \tau, d y^{\prime}\right) q(\tau, y) \\
\quad & \times \int_{R} \pi\left(y^{\prime} \mid \tau, y, d z\right) P\left(\left(\tau, r_{2}, \cdots, r_{n}\right), \tilde{b}\left(z, y_{2}, \cdots, y_{n}\right), t, E\right),
\end{aligned}
$$

where $\hat{r}=\left(r_{2}, \cdots, r_{k}\right)$ are time parameters which correspond to the closest subbranch $\hat{b}$ of $\tilde{b}$ to the first end point, $s_{1}=\left(s_{1}, \ldots,{ }^{s} \sharp\left(b_{1}\right)\right)$ and $\left.s_{2}=\left(s_{\#\left(b_{1}\right)+1}, \cdots, s_{\#\left(b_{1}\right)+\#\left(b_{2}\right)}\right) .{ }^{4}\right)$

Outline of the proof. When $\widetilde{b}=b_{0}$, (5) coincides with (3). When $\tilde{b}=\left(b^{\prime}, b^{\prime \prime}\right)$, assume (5) with $\widetilde{b}$ replaced by $b^{\prime}$. Since $\hat{b}$ is also the closest subbranch of $b^{\prime}$ to the first end point, $P\left(\left(s, r_{2}, \cdots, r_{n}\right), \widetilde{b}\left(b(\boldsymbol{x}), y_{2}, \cdots, y_{n}\right)\right.$, $t, E$ ) is equal to

3) These correspondences are of the form $b^{\prime} \times b^{\prime \prime} \rightarrow\left(b^{\prime}, b^{\prime \prime}\right)$.

4) The substitution can take place at any end point of $\widetilde{b}$, where the corresponding formulation is clear. 


$$
\begin{aligned}
& P\left(\left(\boldsymbol{s}, \boldsymbol{r}^{\prime}, \boldsymbol{r}^{\prime \prime}\right),\left(b^{\prime}(b(\boldsymbol{x}), y), b^{\prime \prime}\left(\boldsymbol{y}^{\prime \prime}\right)\right), t, E\right) \\
& =\int_{\max \left(\boldsymbol{s}, \boldsymbol{r}^{\prime}, \boldsymbol{r}^{\prime \prime}\right)}^{t} d \sigma \int_{R^{2}} P\left(\left(\boldsymbol{s}, \boldsymbol{r}^{\prime}\right), b^{\prime}\left(b(\boldsymbol{x}), \boldsymbol{y}^{\prime}\right), \sigma, d y\right) P\left(\boldsymbol{r}^{\prime \prime}, b^{\prime \prime}\left(\boldsymbol{y}^{\prime \prime}\right), \sigma, d y^{\prime}\right) \\
& \quad \times q(\sigma, y) \int_{R} \pi\left(y^{\prime} \mid \sigma, y, d z\right) p_{0}(\sigma, z, t, E) \\
& =\int_{\max \left(\boldsymbol{s}, \boldsymbol{r}^{\prime}, \boldsymbol{r}^{\prime \prime}\right)}^{t} d \sigma \int_{R^{2}}\left\{\int_{\max (\boldsymbol{s}, \hat{\boldsymbol{r}})}^{\sigma} d \tau \int_{R^{2}} P\left(\boldsymbol{s}_{1}, b_{1}\left(\boldsymbol{x}_{1}\right), \tau, d v\right)\right. \\
& \quad \times P\left(\boldsymbol{s}_{2}, b_{2}\left(\boldsymbol{x}_{2}\right), \tau, d v^{\prime}\right) q(\tau, v) \int_{R} \pi\left(v^{\prime} \mid \tau, v, d w\right) \\
& \left.\quad \times P\left(\left(\tau, \boldsymbol{r}^{\prime}\right), b^{\prime}\left(w, \boldsymbol{y}^{\prime}\right), \sigma, d y\right)\right\} P\left(\boldsymbol{r}^{\prime \prime}, b^{\prime \prime}\left(\boldsymbol{y}^{\prime \prime}\right), \sigma, d y^{\prime}\right) q(\sigma, y) \int_{R} \\
& \quad \times \pi\left(y^{\prime} \mid \sigma, y, d z\right) P_{0}(\sigma, z, t, E)
\end{aligned}
$$

with obvious notations $\boldsymbol{r}^{\prime}, \boldsymbol{r}^{\prime \prime}, \boldsymbol{y}^{\prime}, \boldsymbol{y}^{\prime \prime}$. But, this coincides with the right side of (5) by changing the order of integration by $d \sigma$ and $d \tau$, using (3).

Let $\varphi(\boldsymbol{x})$ be the sum of non-negative functions $\varphi_{k}\left(\boldsymbol{x}_{k}\right), k=1,2, \cdots$, measurable in $x_{k}=\left(x_{i_{1}, k}, \cdots, x_{i_{n_{k}, k}}\right)$, and let $I\left(\boldsymbol{x}_{k}\right)$ be the set of indices for $\boldsymbol{x}_{k}$. For a subset $J$ of $I=\{1,2, \cdots\}$, we write

$$
\begin{gathered}
\int f^{\infty} \varphi(\boldsymbol{x})=\sum_{k=1}^{\infty} \int_{R \sharp\left(I\left(\boldsymbol{x}_{k}\right)\right)} \prod_{i \in I\left(\boldsymbol{x}_{k}\right)} f\left(d x_{i}\right) \varphi_{k}\left(\boldsymbol{x}_{k}\right), \\
\int_{J c} f^{\infty} \varphi(\boldsymbol{x})=\sum_{k=1}^{\infty} \int_{R \sharp\left(I\left(\boldsymbol{x}_{k}\right) \cap J^{c}\right)} \prod_{i \in I\left(\boldsymbol{x}_{k}\right) \cap J^{c}} f\left(d x_{i}\right) \varphi_{k}\left(\boldsymbol{x}_{k}\right) .^{5)}
\end{gathered}
$$

Then, by a similar induction as in II of [3], we have

Theorem 3. The minimal solution $P^{(f)}(s, x, t, E)$ of (1) is given by

$$
\begin{aligned}
& P^{(f)}\left(s, x_{1}, t, E\right)=\int_{\{1\} c} f^{\infty} \sum_{b \in T} P((s, \cdots, s), b(x), t, E), \\
& P_{s, t}^{(f)}(E)=\int f^{\infty} \sum_{b \in T} P((s, \cdots, s), b(\boldsymbol{x}), t, E) .
\end{aligned}
$$

3. Applications, a) Chapman-Kolmogorov equation:

( 7$) \quad P^{(f)}(s, x, u, E)=\int_{R} P^{(f)}(s, x, t, d y) P^{\left(P_{s, t}^{(f)}\right)}(t, y, u, E), s \leq t \leq u$.

Proof. By (4) and (6), $P^{(f)}\left(s, x_{1}, u, E\right)$ is equal to

$$
\begin{aligned}
\int_{[1] c} f^{\infty} & \sum_{b \in T} \sum_{b^{\prime} \leq b} \int_{R \sharp\left(b^{\prime}\right)} \prod_{b_{k} \in b_{/ b^{\prime}}} P\left(s, b_{k}\left(\boldsymbol{x}_{k}\right), t, d y_{k}\right) P\left(t, b^{\prime}(\boldsymbol{y}), u, E\right)^{6)} \\
= & \int_{\{1\} c} f^{\infty} \sum_{b^{\prime} \in T} \int_{R^{\sharp\left(b^{\prime}\right)}} \prod_{k=1}^{\left.\# b^{\prime}\right)} \sum_{b k \in T} P\left(s, b_{k}\left(\boldsymbol{x}_{k}\right), t, d y_{k}\right) P\left(t, b^{\prime}(\boldsymbol{y}), u, E\right) \\
= & \sum_{b^{\prime} \in T} \int_{R^{\sharp\left(b^{\prime}\right)}}\left\{\int_{\{1\} c} f^{\infty} \sum_{b_{1} \in T} P\left(s, b_{1}\left(\boldsymbol{x}_{1}\right), t, d y_{1}\right)\right\} \prod_{k=2}^{\#\left(b^{\prime}\right)} \\
& \times\left\{\int^{\infty} f_{b_{k} \in T} P\left(s, b_{k}\left(\boldsymbol{x}_{k}\right), t, d y_{k}\right)\right\} P\left(t, b^{\prime}(\boldsymbol{y}), u, E\right) \\
= & \int_{R} P^{(f)}\left(s, x_{1}, t, d y_{1}\right) \sum_{b^{\prime} \in T} \int_{R \#\left(b^{\prime}\right)-1} \prod_{k=2}^{\#\left(b^{\prime}\right)} P_{s, t}^{(f)}\left(d y_{k}\right) P\left(t, b^{\prime}(\boldsymbol{y}), u, E\right)
\end{aligned}
$$

5) When $f$ is a probability measure, these are the integrals by infinite direct products of $f$ 's.

6) $P(s, b(x), t, E)$ is an abbreviation for $P((s, \cdots, s), b(x), t, E)$. 


$$
=\int_{R} P^{(f)}\left(s, x_{1}, t, d y_{1}\right) \int_{\{1\} c}\left(P_{s, t}^{(f)}\right)^{\infty} \sum_{b^{\prime} \in T} P\left(t, b^{\prime}(\boldsymbol{y}), u, E\right),
$$

coinciding with the right side of (7) by (6).

b) Backward equation (2) for the minimal solution is proved by rewriting

$$
P^{(f)}(s, r, x, t, E)=\int_{\{1\} c} f^{\infty} \sum_{b \in T} P((s, r, r, \cdots, r), b(x), t, E), \quad r, s \leq t,{ }^{7}
$$

in two ways. First, noting that $b_{1} \times b_{2} \rightarrow b_{1}\left(\left(b_{0}, b_{2}\right) b_{0}, \cdots, b_{0}\right)$ is a one to one correspondence between $T \times T$ and $T-\left\{b_{0}\right\}$, and using (5), we have

$$
\begin{aligned}
P^{(f)}(s, & \left., x_{1}, t, E\right)=\int_{\{1\} c} f^{\infty}\left\{P\left(s, b_{0}\left(x_{1}\right), t, E\right)+\sum_{b \in T^{\prime}-\left\{b_{0}\right\}}\right. \\
& \times P((s, r, \cdots, r), b(\boldsymbol{x}), t, E)\} \\
= & P_{0}\left(s, x_{1}, t, E\right)+\int_{\{1\}} f^{\infty} \sum_{b_{1} \in T} \sum_{b_{2} \in T} P\left((s, r, \cdots, r), b_{1}\left(\left(b_{0}\left(x_{1}\right),\right.\right.\right. \\
& \left.\left.\times b_{2}\left(\boldsymbol{x}^{\prime}\right), \boldsymbol{x}^{\prime \prime}\right), t, E\right) \\
= & P_{0}\left(s, x_{1}, t, E\right)+\int_{\{1\}} f^{\infty} \sum_{b_{1} \in T} \sum_{b_{2} \in T} \int_{s \vee r}^{t} d \tau \int_{R^{2}} P\left(s, b_{0}\left(x_{1}\right), \tau, d y\right) \\
& \times P\left((r, \cdots, r), b_{2}\left(\boldsymbol{x}^{\prime}\right), \tau, d y^{\prime}\right) q(\tau, y) \int_{R} \pi\left(y^{\prime} \mid \tau, y, d z\right) \\
& \times P\left((\tau, r, \cdots, r), b_{1}\left(z, \boldsymbol{x}^{\prime \prime}\right), t, E\right) \\
= & P_{0}\left(s, x_{1}, t, E\right)+\int_{s \vee r}^{t} d \tau \int_{R^{2}} P_{0}\left(s, x_{1}, \tau, d y\right) P_{r, \tau}^{(f)}\left(d y^{\prime}\right) q(\tau, y) \int_{R} \\
& \times \pi\left(y^{\prime} \mid \tau, y, d z\right) P^{(f)}(\tau, r, z, t, E) .{ }^{8)}
\end{aligned}
$$

On the other hand, we can prove, by (4),

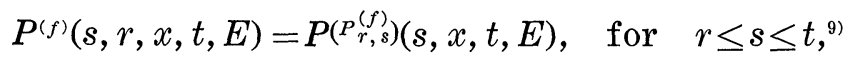

and hence (2) is obtained by substituting (10) into the left and the right extremes of (9) with $r$ replaced by $s_{0}$. In fact, $P^{(f)}(s, r, x, t, E)$ is equal to

$$
\begin{gathered}
\int_{\{1\}^{c}} f^{\infty} \sum_{b \in T^{\prime}} \sum_{b^{\prime} \leq b} \int_{R_{\sharp}\left(b^{\prime}\right)} P\left((s, r, \cdots, r), b_{1}\left(\boldsymbol{x}_{1}\right), s, d y_{1}\right) \prod_{\substack{\left(b k \in b / b^{\prime}\right) \\
k \geq 2}} \\
P\left(r, b_{k}\left(\boldsymbol{x}_{k}\right), s, d y_{k}\right) P\left(s, b^{\prime}(\boldsymbol{y}), t, E\right)
\end{gathered}
$$

(where $b_{1}$ is the first of $b / b^{\prime}$ )

7) Intuitively, this is the probability that the particle, started at $x_{1}$ at time $s_{1}$, is in the set $E$ at time $t$ after the interactions governed by $b$ with other particles which started at time $r$ with the common initial distribution $f$ independently. Clearly, this reduces to $P^{(f)}(s, x, t, E)$ when $r=s$.

8) The corresponding equation of forward type is

$$
\begin{aligned}
P^{(f)} & (s, r, x, t, E)=P_{0}(s, x, t, E)+\int_{s \vee r}^{t} d \tau \int_{R^{2}} P^{(f)}(s, r, x, \tau, d y \\
& \times P_{r, \tau}^{(f)}\left(d y^{\prime}\right) q(\tau, y) \int_{R} \pi\left(y^{\prime} \mid \tau, y, d z\right) P_{0}(\tau, z, t, E) .
\end{aligned}
$$

This is proved in a similar way, or by a successive approximation similar to the proof of (6). Note that this reduces to (1) when $r=s$.

9) In case $s \leq r \leq t, P^{(f)}(s, r, x, t, E)=\int_{R} P_{0}(s, x, r, d y) P^{(f)}(r, y, t, E)$. 


$$
\begin{aligned}
= & \int_{\{1\} c} f^{\infty} \sum_{b^{\prime} \in T} \int_{R \#\left(b^{\prime}\right)} \sum_{b_{1} \in T} P\left((s, r, \cdots, r), b_{1}\left(\boldsymbol{x}_{1}\right), s, d y_{1}\right) \prod_{k=2}^{\#\left(b^{\prime}\right)} \\
& \times \sum_{b k \in T} P\left(r, b_{k}\left(\boldsymbol{x}_{k}\right), s, d y_{k}\right) P\left(s, b^{\prime}(\boldsymbol{y}), t, E\right) \\
= & \sum_{b^{\prime} \in T} \int_{R \#\left(b^{\prime}\right)} \delta x_{1}\left(d y_{1}\right) \prod_{k=2}^{\#\left(b^{\prime}\right)} P_{r, s}^{(f)}\left(d y_{k}\right) P\left(s, b^{\prime}(\boldsymbol{y}), t, E\right)=P^{\left(P_{r}^{(f)}\right)}\left(s, x_{1}, t, E\right),
\end{aligned}
$$

since $P((s, r, \cdots, r), b(\boldsymbol{x}), s, E)=\delta_{x_{1}}(E)$ or 0 according as $b=b_{0}$ or not for $r \leq s$.

4. Let $b^{\prime} \leq b$ and define a substochastic measure on $\left(R^{\sharp\left(b^{\prime}\right)}, \mathcal{B}\left(R^{\sharp\left(b^{\prime}\right)}\right)\right)$ by

where

$$
P\left(b / b^{\prime}, \boldsymbol{s}, \boldsymbol{x}, t, d \boldsymbol{y}\right)=\prod_{b_{k} \in b / b^{\prime}} P\left(s_{k}, b_{k}\left(\boldsymbol{x}_{k}\right), t, d y_{k}\right),
$$

Then, the following extension of (4) is proved easily.

$$
\begin{aligned}
& P\left(b / b^{\prime}, \boldsymbol{s}, \boldsymbol{x}, u, E\right)=\sum_{b^{\prime} \leq b^{\prime \prime} \leq b} \int_{R \#\left(b^{\prime \prime}\right)} P\left(b / b^{\prime \prime}, s, \boldsymbol{x}, t, d \boldsymbol{y}\right) \\
& \quad \times P\left(b^{\prime \prime} / b^{\prime}, t, \boldsymbol{y}, u, E\right)^{11)}
\end{aligned}
$$

Let $b \geq b_{1} \geq b_{2} \geq \cdots \geq b_{n}, t_{0} \leq t_{1} \leq \cdots \leq t_{n}, E_{1} \in \mathcal{B}\left(R^{\sharp\left(b_{1}\right)}\right), \cdots, E_{n}$ $\in \mathscr{B}\left(R^{\sharp\left(b_{n}\right)}\right)$, and let

$$
\begin{aligned}
P\left(t_{0},\right. & \left.t_{1}, \cdots, t_{n} ; b, b_{1}, \cdots, b_{n} ; \boldsymbol{x}, E_{1}, \cdots, E_{n}\right)=\int_{E_{1}} P\left(b / b_{1}, t_{0}, \boldsymbol{x}, t_{1}, d \boldsymbol{x}_{1}\right) \int_{E_{2}} \\
\quad \times & P\left(b^{1} / b_{2}, t_{1}, \boldsymbol{x}_{1}, t_{2}, d \boldsymbol{x}_{2}\right) \cdots \int_{E_{n-1}} P\left(b_{n-2} / b_{n-1}, t_{n-2}, \boldsymbol{x}_{n-2}, t_{n-1}, d \boldsymbol{x}_{n-1}\right) \\
\quad \times & P\left(b_{n-1} / b_{n}, t_{n-1}, \boldsymbol{x}_{n-1}, t_{n}, E_{n}\right) .
\end{aligned}
$$

Then, a version of the consistency condition holds :

$$
\begin{aligned}
& P\left(t_{0}, t_{1}, t_{3}, \cdots, t_{n} ; b_{0}, b_{1}, b_{3}, \cdots, b_{n} ; \boldsymbol{x}, E_{1}, E_{3}, \cdots, E_{n}\right) \\
& =\sum_{b_{1} \geq b_{2} \geq b_{3}} P\left(t_{0}, t_{1}, t_{2}, t_{3}, \cdots, t_{n} ; b_{0}, b_{1}, b_{2}, \cdots, b_{n} ; \boldsymbol{x}, E_{1}, R^{\sharp\left(b_{2}\right)} E_{3}, \cdots, E_{n}\right),
\end{aligned}
$$

where we skipped $t_{2}$ alone for simplicity. This suggests that (11) is the probability of a cylinder set of a probability space which describes all interactions suffered by the particular particle we are watching at.

\section{References}

[1] W. Feller: On the integro-differential equations of purely discontinuous Markov processes. Trans. Amer. Math. Soc., 48, 488-515 (1940) [erratum, 58, 474 (1945)].

[2] T. Ueno: A class of Markov processes with bounded, non-linear generators. Jap. J. Math., 38, 19-38 (1969).

[3] - : A class of purely discontinuous Markov processes with interactions. I, II. Proc. Japan Acad., 45, 348-353, 437-440 (1969).

[4] - : A class of Markov processes with interactions. I. Proc. Japan Acad., 45, 348-353 (1969).

10) Since $b / b_{0}=\{b\}, P\left(b / b_{0}, s, x, t, E\right)=P(s, b(x), t, E)$ and (4') reduces to (4) in case $b^{\prime}=b_{0}$.

11) $P\left(b / b^{\prime}, s, x, t, E\right)$ is an abbreviation for $P\left(b / b^{\prime},(s, \cdots, s), x, t, E\right)$. 